\title{
Similarity comparisons with remembered and perceived magnitudes: Memory psychophysics and fundamental measurement
}

\author{
WILLIAM M. PETRUSIC \\ Carleton University, Ottawa, Ontario, Canada \\ JOSEPH V. BARANSKI \\ Defense and Civil Institute of Environmental Medicine, North York, Ontario, Canada \\ and \\ RUTH KENNEDY \\ Carleton University, Ottawa, Ontario, Canada
}

\begin{abstract}
At the outset, subjects learned to associate a label with each element in a set of perceptual magnitudes (visual extents), using traditional paired-associate learning methods. Subsequently, on some trials, subjects indicated which pair of two pairs of labels corresponded to the more similar perceptual referents, and, on other trials, they selected the more dissimilar pair. It is shown that these similarity comparisons satisfy the axioms (transitivity and intradimensional subtractivity) necessary to conclude that they are based on computation of the difference of the differences of analogue-based interval scale representations. The findings also permitted refutation of the idea that memory for elementary percepts arises from their reperception. Notably, the memory exponent was 0.697 , but the perception exponent was 0.546 , and the reperception idea requires that the memory exponent be the square of the perception exponent $\left(0.546^{2}=0.298\right)$. Symbolic distance effects and enhanced response time-based semantic congruity effects, typically found with binary comparisons, extend the range of commonalties found between perceptual and memory psychophysics.
\end{abstract}

\begin{abstract}
Memory psychophysics extends Fechner's (1860/1966) outer psychophysics to a determination of the quantitative relationship between the physical magnitude of a stimulus and its subjective magnitude-not as it is perceived but, rather, as it is remembered. In this article, we explore the properties of the representation of long-term memory for elementary visual percepts and the representation of the percept itself, and we examine their interrelationships in detail. We begin with a brief review of the principal findings based on the application of classical psychophysical techniques to remembered stimuli, and we discuss some of the limitations of that work. We then address the fundamental question of whether a memorial
\end{abstract}

This research was supported by a grant from the Natural Sciences and Engineering Research Council of Canada to W.M.P. Portions of this work were presented at the XXV International Congress of Psychology, Brussels, July, 1992. The experiment was conducted by R.K. as part of her master's degree requirements at the Department of Psychology, Carleton University. Our thanks to Geoffrey Loftus, Gregory Lockhead, and two anonymous reviewers for their helpful comments. Correspondence should be addressed to W. M. Petrusic, Department of Psychology, Carleton University, Colonel By Drive, Ottawa, Ontario, K1S 5B6, Canada (e-mail: bill_petrusic@carleton.ca). representation of a basic element of sensory experience can be maintained in memory at the level of an interval scale, by extending the axiomatic approach of fundamental measurement (Krantz, Luce, Suppes, \& Tversky, 1971) to the domain of memory psychophysics by requiring comparative judgments of similarity/dissimilarity. Specifically, the subjects were presented with two pairs of pairs (quads) and were required, on some trials, to indicate which pair was more similar and, on other trials, which was more dissimilar. Because binary comparisons were required with two pairs of pairs, as distinguished from but a single pair, these judgments are referred to as quaternary relational judgments. In order to obtain quaternary relational judgments and thereby determine whether interval scale representations (i.e., analogue representations) could be obtained in memory, the subjects first learned to associate labels with horizontal lines varying in extent, and subsequently they were required to indicate in which of the two pairs the labels representing the referent line lengths were more similar/dissimilar. Finally, we complement the fundamental measurement approach with an analysis of response times (RTs) in an attempt to characterize the nature of the decision process for quaternary relational judgments with perceived and remembered magnitudes. 


\section{MEMORY PSYCHOPHYSICS: ISSUES AND TECHNIQUES}

The principal aim of the program of memory psychophysics is to provide a quantitative characterization of memories of perceptual experiences. Although this objective has been addressed in various ways (see Algom, 1992; Baranski \& Petrusic, 1992), the main foci of contemporary research in (unidimensional) memory psychophysics have been to determine (1) the quantitative relation between subjective estimates of perceived and those of remembered magnitudes, and (2) the metric properties (i.e., scale type) of the representation of remembered magnitudes - namely, is the scale at an ordinal or an interval level of measurement? We consider these in turn.

\section{The Psychophysical Function for Memory}

Ratio scaling. Using direct ratio-scaling methods (see Stevens, 1975), Björkman, Lundberg, and Tarnblom (1960) made the first attempt to determine the properties of the psychophysical function for remembered magnitudes and its relationship to the psychophysical function obtained when stimuli are perceived. In the first phase of the Björkman et al. experiments, observers studied a set of circles varying in area in one experiment and cylinders varying in weight in another and, through pairedassociate training, learned to associate labels with each stimulus. In one experiment, subjects judged the ratio between the magnitude of a remembered stimulus and the magnitude of a perceptually available stimulus. In other experiments, subjects directly estimated the magnitude of both perceived and remembered stimuli. Their findings demonstrated that the relation between the perceived and remembered subjective magnitudes was related to the underlying physical magnitudes by a power function. However, the results were rendered somewhat equivocal by the fact that judgments were required of the perceived and remembered magnitudes at the same time.

Magnitude estimation: Memory for elementary sensory magnitudes as reperception. Extending Björkman et al.'s (1960) methodology, Moyer, Bradley, Sorensen, Whiting, and Mansfield (1978) and Kerst and Howard (1978) demonstrated that numerical magnitude estimates of the perceived and remembered sizes of objects were well described by power functions of their physical sizes. Noting that the value of the exponent of the power function for memory was approximately equal to the value of the perception exponent squared, they formulated the reperception hypothesis. According to this view, the remembered representation arises upon applying a power transformation to the perceived magnitude, which in turn arises as a power function of the physical magnitude. Formally, the psychophysical function for perception is given by $\psi_{P}(x)=\alpha_{P} x^{\beta_{P}}$, where $\alpha_{P}$ and $\beta_{P}$, respectively, denote the unit of measurement and the exponent, and $\psi_{P}(x)$ denotes the perceptual magnitude of a stimulus with physical magnitude $x$. Because the input to memory is a power function of the perceptual magnitude, it follows that $\psi_{M}(x)=\alpha \psi_{P}(x)^{\beta}$, where $\beta$ denotes the exponent for the transformation from perception to memory, and $\psi_{M}(x)$ denotes the subjective magnitude in memory.

According to the reperception hypothesis, as it has been invariably expressed (e.g., Kerst \& Howard, 1978; Moyer et al., 1978; and see Algom, 1992, for a review), $\beta=\beta_{P}$. Consequently, the memory psychophysical function is given by $\psi_{M}(x)=\alpha_{M} x \beta_{M}$, with $\alpha_{M}=\alpha \alpha_{P}$ and $\beta_{M}$ $=\beta^{2}$.

Hence, because the exponent for the memory psychophysical function is the square of the exponent of the perceptual psychophysical function, if the perceptual exponent is compressive (i.e., $<1$ ), the memorial exponent should be more compressive. Conversely, if the perceptual exponent is expansive (i.e., $>1$ ), the memorial exponent should be more expansive. Alternatively, and slightly more generally, the reperception hypothesis asserts that if $\beta_{P}>1$, then $\beta_{M}>\beta_{P}$, and if $\beta_{P}<1$, then $\beta_{M}<\beta_{P}$.

Of course, alternative expressions of the reperception idea are possible. For example, the memory transformation could be uniformly expansive - that is, $\beta>1$. Consequently, the memory exponent would be always greater than the perceptual exponent. However, the available data permit clear rejection of this case, because memory exponents are very frequently less than their perceptual counterparts (see Algom, 1992). Similarly, the memory transformation exponent, $\beta$, could always be less than one - that is, uniformly compressive. ${ }^{1}$ In this case, the memory exponent always would be less than the perception exponent. However, the available findings also permit the rejection of the notion that memory is uniformly compressive, because there are instances in which exponents in memory are larger than those in perception (e.g., roughness; Moyer, Sklarew, \& Whiting, 1982). Thus, the reperception idea has come to be identified with the special case of $\beta=\beta_{p}$.

To date, a number of studies have provided clear support for the reperception hypothesis with $\beta=\beta_{P}$ (see, e.g., Bradley \& Vido, 1984; Chew \& Richardson, 1980; Kerst \& Howard, 1978; Moyer et al., 1978), whereas other studies have not (see Algom, 1992). As an example of the latter, Moyer et al. (1982) showed that, when $\beta_{P}>1$ (e.g., heaviness and sweetness), the memory exponent is less than the perceptual exponent, although it should be larger than it.

Unfortunately, when direct magnitude estimation methods are used, it is not clear whether the violations of the reperception idea arise from its basic failure or because of inherent difficulties with the provision of numerical estimates that purportedly preserve ratios of sensation magnitudes (see Krueger, 1989, concerning the representation of number and its role in determining the exponent in the power function). Consequently, we propose that it would be desirable to reexamine the reperception idea when the problems arising from direct numerical estimation are circumvented. 
The Memory Metric:

\section{Response Times for Binary Comparisons}

The chronometric approach to contemporary memory psychophysics began with Moyer's (1973) landmark experiment requiring subjects to compare the sizes of animals from memory. Soon after, Moyer and Bayer (1976) addressed the fundamental question of the memory metric. In the first phase of their experiment, subjects learned to associate nonsense syllables (CVCs) with each of four circles varying in size. For one group of subjects, the circles spanned a wide range, and, for another group, the range was narrow (although both groups could readily learn to associate the labels with each stimulus). Moyer and Bayer found that, as expected, RTs for comparing perceptual stimuli (i.e., circle-circle) were slower for the narrow range group. More importantly, they found that RTs for the remembered comparisons (i.e., CVC-CVC) also were slower for the narrow range group. They concluded that this result could arise only if interval scale information was preserved in memory. In fact, as will become evident in the sequel, their finding did not serve to establish unequivocally an interval scale for remembered magnitudes; rather, it was merely sufficient to deny that the representation was exclusively ordinal, as predicted on the basis of the currently popular propositionally based discrete semantic-coding theories (e.g., Banks, 1977; Banks, Mermelstein, \& Yu, 1982; Cech \& Shoben, 1985; Cech, Shoben, \& Love, 1990). Indeed, the Moyer and Bayer range effect suggests that the representation is at least at the level of an ordered-metric scale. Ordered-metric scales arise when in addition to an ordering of the stimuli, an ordering of differences is obtained. Although these scales are "not quite interval scales" (see Krantz, Luce, Suppes, \& Tversky, 1971, pp. 430-431) nonmetric scaling (Kruskal, 1964; Shepard, 1966) applied to the ordering of intervals permits recovery of a representation.

Although the Moyer and Bayer (1976) range effect was sufficient to implicate a memory representation more tightly constrained than a mere ordinal scaling, its empirical status to date remains equivocal. On the one hand, two attempts to replicate the range effect have proved unsuccessful (Banks et al., 1982; Henderson \& Well, 1985). On the other hand, the Banks et al. study also failed to obtain evidence of a perceptual range effect, which is a necessary condition for its occurrence in memory; Henderson and Well did not determine whether the perceptual range effect was evident in their experiment. More recently, Petrusic, Baranski, and Aubin (1995) found marginally reliable range effects for both perception and memory, and they discuss a number of methodological and statistical constraints on observing the RT range effect.

In sum, a clear interpretation of some of the critical issues in unidimensional memory psychophysics research has been complicated by methodological problems with magnitude estimation techniques and equivocal results in chronometric analyses attempting to determine the metric scale of remembered magnitudes. In the present paper, we examine simultaneously the metric of remembered magnitudes and the status of the memorial psychophysical function, while overcoming the aforementioned methodological difficulties. In doing so, we hope to fill a void in contemporary memory psychophysics research that was first noted some 15 years ago by Nelson and Chaiklin (1980).

What is required is an empirical test of a set of axioms that has been proven to yield an interval scale in the style of measurement theory (see Krantz, Luce, Suppes, \& Tversky, 1971). However, since an empirical test of such a set of axioms has not yet occurred, there is no compelling evidence for an interval scale of the psychological conception of space. (p. 530)

\section{FUNDAMENTAL MEASUREMENT AND MEMORY PSYCHOPHYSICS}

Similarity/dissimilarity scaling methods, developed for the direct comparison of intervals, provide an alternative and largely unexplored set of psychophysical methods with which to determine the metric properties of memory representations. The landmark use of similarity comparison data and multidimensional scaling (MDS) algorithms led Shepard and Chipman (1970) to propose the concept of second-order isomorphism through their analyses of rankings of perceived and remembered shapes of 15 American states. However, although their analyses established that the two-dimensional representations of the shapes of the states were quite similar for memory and perception, it remains unclear whether in fact the conditions necessary for such a representation were well satisfied. For example, one such condition-requiring that subjects are able to order the pairs of states in terms of shape-requires examination of the transitivity axiom. Because Shepard and Chipman's task required a rank ordering of the pairs, transitivity was not tested (quite reasonably; with 105 pairs of states, 5,460 pairs of pairs [105 $\times 104 / 2]$ would have to be compared to test transitivity); rather, it was imposed. In the experiment to be reported, transitivity was not imposed; it was explicitly tested, along with the other testable axioms for a positive difference structure.

\section{Positive-Difference Structures}

Elegant and highly developed quantitative theories exist, in the form of a set of axioms, that permit a mathematical proof of the existence of a representation that preserves the observable properties of the empirical relation on which the representation is based. In the present case, the empirical relation, denoted $\boldsymbol{S}$, arises from a direct comparison of two pairs of stimuli, $a b$ and $c d$, for example; whenever the subject judges the pair $a b$ as more similar than the pair $c d$, we say the pairs $a b$ and $c d$ are in the (quaternary) relation $\boldsymbol{S}$ and we denote this by $a b \boldsymbol{S} c d .^{2}$ The fundamental measurement approach imposes con- 
straints, in the form of axioms, on the relation $S$ (i.e., on the similarity comparisons) such that, if these constraints are satisfied, it is possible to obtain an interval scale representation of the stimuli from the similarity-based ordering of the stimulus pairs. The word positive in the expression positive-difference structure arises from the fact that the representation of sensory or memory differences is in terms of positive intervals. Positivity is readily ensured through the use of a set of stimuli, such as extent, for which subjects are easily able to order the underlying elements - that is, the extents that will be employed in the direct comparisons of pairs of pairs for similarity can be clearly distinguished in terms of their lengths.

The representation for a positive-difference structure is given by

$$
a b S c d \text { iff } s(a)-s(b) \leq s(c)-s(d),
$$

where $s(a)$ denotes the representation of stimulus $a-$ that is, the pair $a b$ is judged as more similar than the pair $c d$ whenever the difference in the representations of stimuli $a$ and $b$ is less than the difference in the representations of stimuli $c$ and $d$, and conversely.

The critical, testable axioms for a positive-difference structure (see Krantz et al., 1971, for the formal treatment of difference measurement, and Beals, Krantz, \& Tversky, 1968 , and Block \& Marschak, 1960, for axiomatizations of closely related systems) can be stated as follows.

Weak ordering requires (1) comparability (i.e., the subject can render a judgment) and (2) transitivity, which is defined as, for all triples of pairs $a b, c d, e f$,

if $a b \boldsymbol{S} c d$ and $c d \boldsymbol{S} e f$, then $a b S e f$.

Positivity requires that, if $a b, b c$, and $a c$ are in the set of pairs to be compared, then $a b \boldsymbol{S} a c$ and $b c \boldsymbol{S a c}$. In the present unidimensional context, with clearly distinguishable stimuli, this axiom will be satisfied perfectly and is another way of saying that the stimuli can be ordered, with $a>b>c$, on the underlying sensory (memorial) continuum. ${ }^{3}$

Weak monotonicity, also known as the sextuple condition, is a necessary axiom and is the axiom typically tested. Weak monotonicity, which ensures homogeneous concatenation of intervals throughout the underlying attribute, requires that for all elements $a, b, c, a^{\prime}, b^{\prime}$, and $c^{\prime}$ in the stimulus set,

$$
\text { if } a b \boldsymbol{S} a^{\prime} b^{\prime} \text { and } b c \boldsymbol{S} b^{\prime} c^{\prime} \text {, then } a c \boldsymbol{S} a^{\prime} c^{\prime} \text {. }
$$

Given that these axioms and the technical (i.e., nontestable ${ }^{4}$ ) axioms are satisfied, a positive-difference representation, as specified in (1) above, is guaranteed. Furthermore, this representation is unique up to a linear transformation, thus ensuring an interval scale representation- - that is, if $s^{\prime}(x)$ and $s(x)$ are any two representations satisfying the axioms, then $s^{\prime}(x)=a s(x)+b$, where $a>0$ and $b$ are constants, reflecting changes in the unit of measurement and the origin of the scale, respectively.
In alternative axiomatizations of difference systems (Block \& Marschak, 1960; Suppes \& Winet, 1955; Suppes \& Zinnes, 1963), the quadruple condition, which we refer to as intradimensional subtractivity (IDS), is used in place of the monotonicity axiom. IDS is defined as: for all subsets of stimuli $a, b, c$, and $d$, with $a>b>c>d$,

$$
a b \boldsymbol{S} c d \text { iff } a c \boldsymbol{S} b d \text {. }
$$

Fuller appreciation of the IDS axiom is evident on noting that, given the representation exists, then $a c S b d$ iff $s(a)-s(c)=s(a)-s(b)+s(b)-s(c)<s(b)-s(d)=$ $s(b)-s(c)+s(c)-s(d)$, but on canceling the common distance, $s(b)-s(c)$, the inequality reduces to $s(a)-$ $s(b)<s(c)-s(d)$, which is equivalent to $a b S c d$. Thus, comparisons with quads of the form $(a c, b d)$ can be reduced to comparisons of quads of the form $(a b, c d)$ through the cancellation of the common distance $s(b)-$ $s(c)$. We thus say that the IDS axiom is necessary-that is, it follows on assuming the existence of the representation. ${ }^{5}$

\section{Metric Representability and \\ Implications for Memory Psychophysics}

If the representations in memory preserve only the strict ordinal relations among the underlying perceptual referents, as suggested by some propositionally based accounts of symbolic comparisons, it is unlikely that similarity comparisons with symbolic comparisons could be rendered, except for those based on differences in ordinal magnitudes. On the other hand, if the memory representations activated are invariant up to at least a linear transformation (i.e., an interval scale), then, of course, the comparison of two intervals is possible, and the similarity comparison could be rendered. Thus, satisfaction of the major testable axioms (transitivity, monotonicity, and IDS) and consequently obtaining a metric representation of remembered magnitudes would tightly constrain semanticcoding-based theories (see, e.g., Banks, 1977; Banks et al., 1982; Cech \& Shoben, 1985) and force the development of more highly articulated propositional systems capable of the generation of relations among intervals.

\section{Structural Models for Similarity \\ Comparison: Response Time Analyses}

Although comparisons of pairs of pairs for similarity/ dissimilarity have been obtained only rarely (see, e.g., Petrusic \& Jamieson, 1989; Schneider, Parker, \& Stein, 1974), studies of the decision processes involved with such comparisons are even more infrequent. Dember (1957), extending Coombs' $(1952,1964)$ theory of data to an analysis of RTs, required subjects to decide, in one experiment, which of two pairs of brightness patches was more similar and, in another experiment that called for memorybased information, which of two pairs of adjectives was more similar. Dember, in preliminary examination of RTs in this more complex task domain, showed a dependence of RT on the differences of the differences to be com- 
pared. However, Dember's landmark examination excluded quads of the form $(a c, b d)$, where the elements are ordered $a>b>c>d$ on the underlying continuum. Consequently, it was not possible to determine whether the IDS axiom was satisfied.

With a view toward characterizing the decision process involved in similarity/dissimilarity comparisons, we complement the fundamental measurement analyses with an examination of RTs with both perceived and remembered magnitudes. First, we examined the generality of Dember's (1957) conclusion that RTs were consistent with a difference of differences decision rule. According to this rule, RTs with the quad $(a b, c d)$ are a monotonic decreasing function, $F$, of the difference in the distances between the stimuli in each pair-that is,

$$
R T(a b, c d)=F(d(a, b)-d(c, d)),
$$

where $d(a, b)=s(a)-s(b)$ denotes the distance between the representations of stimuli $a$ and $b$ on the underlying continuum. Second, we sought to determine whether semantic congruity effects, to date only evident with binary comparisons, would occur with quaternary relational judgments. Semantic congruity effects would be demonstrated by RT $(a b S c d)<\mathrm{RT}(a b D c d)$ if the pairs $a b$ and $c d$ are similar but by RT $(a b S c d)>\mathrm{RT}(a b D c d)$ if the pairs $a b$ and $c d$ are dissimilar.

In summary, the objective of the present research was to extend the axiomatic approach of fundamental measurement to the domain of unidimensional memory psychophysics. Three major findings will be reported. First, we will demonstrate that quaternary relational judgments with remembered magnitudes satisfy the axioms for a positivedifference structure that is, we confirm an interval scale for remembered magnitudes and then obtain a powerfunction-based representation for each subject separately for perception and for memory by applying a multidimensional scaling routine (ALSCAL) to the obtained rank orderings of distances. Second, we will provide a critical test of the reperception hypothesis of the memorial psychophysical function, while circumventing the problems associated with magnitude estimation techniques. Finally, our RT analyses will demonstrate that formally equivalent decision processes are involved in resolving perceptual and memorially based similarity/dissimilarity judgments.

\section{METHOD}

\section{Subjects}

Eleven psychology graduate students served as paid volunteers for one 2-h session. An additional subject was unable to perform some of the remembered comparisons, and his data were excluded from the analyses.

\footnotetext{
Apparatus

Stimuli were presented on an Amdek-310A video monitor with 720 pixels horizontally and 640 vertically. Graphics production, presentation of instructions and stimuli, event sequencing and timing, and the recording of responses and RTs were controlled by an IBM-PC/XT clone computer. Responses were made by using the buttons on an IBM-PC mouse.
}

\section{Stimuli}

The stimuli were six horizontal line segments: $33,100,200,333$, 466 , and 633 pixels $(10.54,31.95,63.90,106.39,148.88$, and $202.27 \mathrm{~mm}$ ). The lines appeared amber on a black background, were presented at eye level, and were viewed at a distance of approximately $50 \mathrm{~cm}$ in a dimly lit room. The shortest line subtended a visual angle of approximately $1^{\circ}$, and the longest, $23^{\circ}$.

\section{Procedure}

The experiment had two parts: a learning phase and a similarity/dissimilarity judgment phase. Each subject was highly familiar with the set of remembered magnitudes; the subjects had served in two previous 2-h sessions in which they made binary comparisons with six remembered magnitudes (Kennedy, 1990), and each of these sessions was preceded by an acquisition phase that involved extensive overlearning.

Learning phase. The present experiment began by ensuring that the subjects retained the stimulus-CVC label associations acquired in the two sessions preceding the similarity/dissimilarity comparison session. In order to keep the similarity comparison task manageable, we used five of the original six line lengths. Half the subjects worked with the stimulus set 10.54, 31.95, 63.9, 106.39, and $148.88 \mathrm{~mm}$; the other half worked with the set $10.54,31.95,63.9$, 148.88 , and $202.27 \mathrm{~mm}$.

The set of CVCs (e.g., CED, GOZ, JIB, NAD, and WUM) was counterbalanced over subjects and lines. On each learning trial, one of the five lines was presented in the middle of the screen. All five CVCs appeared below the line in individual boxes (in a random order on each trial). By moving the mouse from side to side, the subjects could illuminate any one of the boxes containing the CVCs. The subjects depressed the middle key on the mouse when they assumed the illuminated $\mathrm{CVC}$ to correspond to the line presented on the screen. If they were correct, the CVC remained illuminated for $2 \mathrm{sec}$. If they were incorrect, the correct $\mathrm{CVC}$ was illuminated for $2 \mathrm{sec}$. Subjects had $5 \mathrm{sec}$ to perform the association. If this time was exceeded, Too Slow appeared, and, again, the correct CVC was illuminated for $2 \mathrm{sec}$. The subjects performed learning trials until a criterion of 15 correct matches in a row were obtained (i.e., 3 with each line). This was followed by an additional 15 overlearning trials ( 3 with each line).

Similarity/dissimilarity comparison phase. The two pairs of lines in each quad were centered horizontally and vertically above one another; $20 \mathrm{~mm}$ separated the bottom stimulus of the top pair from the top stimulus of the bottom pair. On perceptual trials, the lines in each pair were separated by $5 \mathrm{~mm}$. On memory trials, the CVCs in each quad appeared in the same vertical locations as the lines.

With five stimuli there are 10 pairs and 55 pairs of pairs. To keep the task manageable, we excluded quads in which no metric information was gained (i.e., quads in which the judgment could be rendered on the basis of merely knowing the ordinal relations among the stimuli). For example, the enveloping quads [e.g., $(a d, b c)]$ and the unilateral quads [e.g., $(a b, a c)$ ], in which the stimuli are ordered $a>b>c>d$, were removed (i.e., it was assumed that the positivity axiom would be perfectly satisfied). Upon removal of these quads, 20 quads remained. After counterbalancing for position (i.e., each pair in each quad and each line $[C V C]$ in each pair appeared equally often on the top and the bottom), a total of 80 combinations of quads was defined. These were each presented twice, once with each instruction (Which is more similar/dissimilar?). resulting in a total of 160 trials per session. Each trial began with the presentation of an instruction (Which is more similar/dissimilar?). The quad appeared $1 \mathrm{sec}$ later. Both the instruction and the quad remained on the screen until the subject responded.

The subjects performed two sessions of similarity/dissimilarity judgments, the first with the remembered stimuli (CVCs) and the second with the perceptual stimuli. Instructions for the similar- 
Table 1

Frequency Distribution of the Number of Violations of Weak Stochastic Transitivity in Memory and in Perception

\begin{tabular}{|c|c|c|}
\hline \multirow{2}{*}{$\begin{array}{l}\text { No. Intransitivities } \\
\text { (Out of } 120 \text { Tests) }\end{array}$} & \multicolumn{2}{|c|}{$\begin{array}{c}\text { No. Subjects } \\
\text { Displaying Intransitivities }\end{array}$} \\
\hline & Memory & Perception \\
\hline 0 & 7 & 7 \\
\hline 1 & 1 & 3 \\
\hline 2 & 2 & 0 \\
\hline 3 & 1 & 0 \\
\hline 4 & 0 & 0 \\
\hline 5 & 0 & 0 \\
\hline 6 & 0 & 1 \\
\hline $\begin{array}{l}\text { Total no. intransitivities } \\
\text { across all subjects }\end{array}$ & 8 out of 1,320 tests & 9 out of 1,320 tests \\
\hline
\end{tabular}

ity/dissimilarity comparison task emphasized accurate responding for both the remembered and the perceptual stimuli.

\section{RESULTS AND DISCUSSION}

\section{Tests of the Axioms for}

\section{a Positive-Difference Structure}

Weak stochastic transitivity (WST). WST, the weakest probabilistic version of the axiom asserting that subjects can order the intervals, requires that

$$
\begin{aligned}
& \text { if } \operatorname{Pr}(a b S c d)>0.50 \text { and } \operatorname{Pr}(c d S e f)>0.50, \\
& \text { then } \operatorname{Pr}(a b S e f)>0.50,
\end{aligned}
$$

where $\operatorname{Pr}(a b S c d)>0.50$ denotes that more than half the time the pair $a b$ was judged more similar than the pair $c d$. For each subject, for each quad, there were four replications with each of the two instructions. Consequently, an estimate of $\operatorname{Pr}(a b S c d)$ was obtained by assuming that $\operatorname{Pr}(a b S c d)=\operatorname{Pr}(c d D a b)$, where $\operatorname{Pr}(c d D a b)$ denotes the probability that stimulus pair $c d$ is more dissimilar than pair $a b$, and then averaging the estimates with the two instructions. Because there were four replications with each instruction, ties could arise. Over all 11 subjects and the 20 quads for each type (perception vs. memory) of comparison, there were seven ties in perception and seven in memory and they were resolved by a coin toss. It was also assumed that judgments with the enveloping and unilateral quads (i.e., those in which the judgment could be rendered by knowing the ordering of the stimuli) would have been made errorlessly.

With five stimuli, there are 10 pairs and 120 triples. Table 1 provides the frequency distribution of the number of triples failing to satisfy WST. Bearing in mind that the expected number of intransitivities for a subject responding by chance is $30(S D=4.74)$, it is clear that the obtained orderings are several standard deviations below chance. Hence, it can be concluded that these subjects are, indeed, capable of ordering intervals, both when the stimuli are perceptually available and when they must be activated in memory.

Intradimensional subtractivity. There were five tests of IDS for each subject. Table 2 provides the frequency distribution of the number of times IDS was satisfied for memory and perception. As Table 2 shows, violations of IDS were rare; they occurred with approximately the same relative frequency in memory as in perception. Violations never occurred when the subjects were perfectly consistent in their choices over the eight replications with either the overlapping or the disjoint quads in both the perception and the memory conditions, suggesting that unreliability in establishing the order relations in each quad was a factor contributing to observing violations of IDS.

Table 2

Frequency Distribution of the Number of Times Intradimensional Subtractivity (IDS) Was Satisfied for Memory and for Perception

\begin{tabular}{ccc} 
No. Times IDS Was & \multicolumn{2}{c}{ No. Subjects Satisfying IDS } \\
\cline { 2 - 3 } Satisfied (Out of Five Tests) & Memory & Perception \\
\hline 0 & 0 & 0 \\
1 & 0 & 0 \\
2 & 0 & 0 \\
3 & 1 & 1 \\
4 & 6 & 7 \\
5 & 4 & 3 \\
was satisfied across all subjects & 47 out of 55 tests & 46 out of 55 tests \\
\hline
\end{tabular}




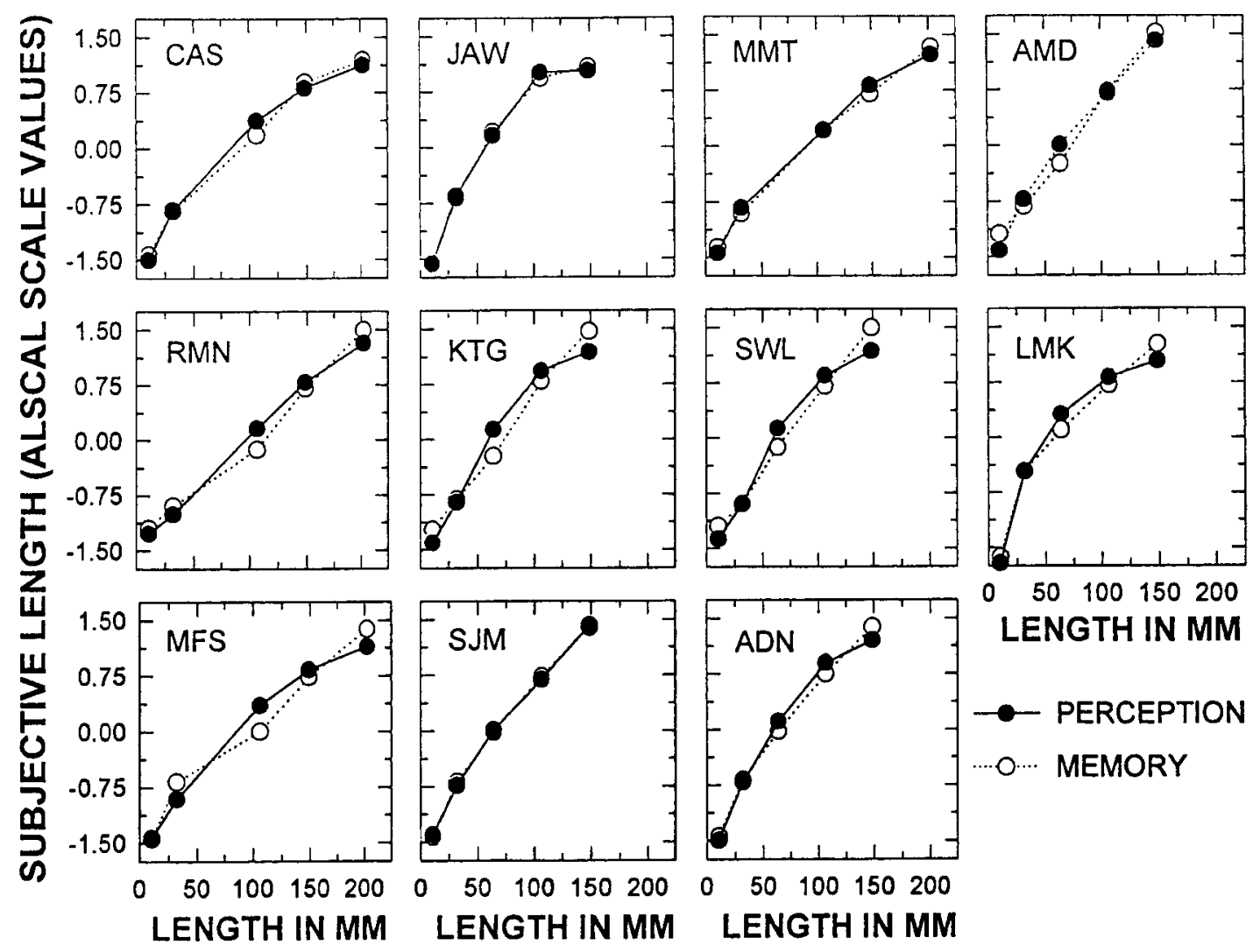

Figure 1. Individual-subject plots of subjective length (ALSCAL scale values) as a function of physical length for perception (filled circles) and memory (unfilled circles).

Hence, there is good support for IDS, and, consequently, metric representations for visual extent can be determined.

\section{Psychophysical Functions for Individual Subjects}

Because intransitivities occurred for some subjects and because the MDS routines require an ordering of intervals, the vote count method was used to resolve intransitivities (see Petrusic \& Jamieson, 1979, 1989, for use of the vote count index in testing axioms for a difference structure). The vote count for stimulus pair $a b$, denoted $V C(a b)$, was obtained by computing $V C(a b)=$ $\sum \operatorname{Pr}(a b S x y)$, where the summation is over all pairs $x y$ excluding $a b$. ALSCAL-Multidimensional Scaling (SPSS Inc., 1988; Takane, Young, \& de Leeuw, 1977) based representations, in one- and in two-dimensional Euclidean space, were obtained for each subject for perception and for memory, using the rank ordering of distances obtained with the vote count index. The stress values with the one-dimensional solutions are acceptably low, in accord with satisfying the axioms for a positive difference structure, ranging from 0.0001 to $0.034(M=0.014, S D=$ 0.012 ) in perception and from 0.0001 to 0.030 in memory $(M=0.011, S D=0.009)$, and they were not appreciably lower for a two-dimensional solution. Figure 1 provides plots of the ALSCAL scale values against the physical extents, separately for perception and for memory. For each subject, the perceptual and memorial functions bear a close resemblance to one another. A more precise examination of this correspondence and its implications for the reperception hypothesis is presented in the next section.

\section{Properties of the Psychophysical Functions}

In order to examine the form of the psychophysical function obtained with the ALSCAL scale values, we assume that the ALSCAL scale value for a stimulus with physical magnitude $x, S(x)$, is linearly related to the representations, $\psi(x)$, in perception and in memory - that is, $\psi(x)=a S(x)+b$, where $a$ and $b$ are multiplicative and additive constants, respectively. Generally, we assume $\psi(x)=$ $a S(x)+b=\alpha x^{\beta}$, with $\alpha>0$, referred to variously as the multiplicative constant and the measure constant, and $\beta>$ 0 , the exponent in the power function. Upon dividing through by $a$ and taking logarithms, $\log (S(x)+b / a)=$ $\log (\alpha / a)+\beta \log (x)$. The value of $\gamma=b / a$ maximizing the correlation between $\log (S(x)+\gamma)$ and $\log (x)$ was then selected, using an iterative computer routine. Given the value of $\gamma$, the exponent, $\beta$, was then obtained by conventional 

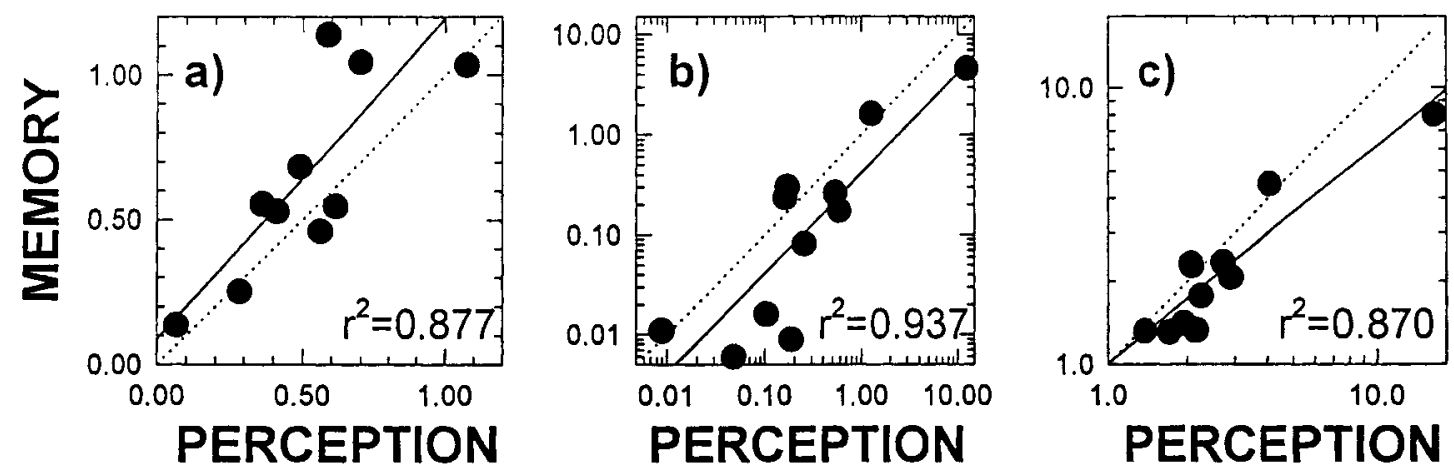

Figure 2. Relation between the memory and the perception power law parameters for the exponent (panel a), multiplicative constant (panel b), and additive constant (panel c). Each point denotes the relation for an individual subject. Solid lines denote the regression line, and dotted lines the main diagonal.

least squares. The procedure used here to fit the power function to ALSCAL scale values parallels that used by Petrusic and Jamieson (1979) and Schneider et al. (1974).

The left panel in Figure 2 provides a plot of the memory exponent as a function of the perception exponent, with each of the 11 subjects contributing a point to the plot. For perception, the mean exponent in the power function is $0.546(S D=0.274$, range $=0.066-1.076)$. For memory, the mean exponent in the power function is 0.697 $(S D=0.376$, range $=0.137-1.283)$. As is evident, there is a strong positive relation between the individual-subject exponents for memory and perception $\left[r^{2}=.677, F(1,9)=\right.$ $18.655, p<.002]$; subjects who show extreme compression in the psychophysical function for perception also show such compression with the memory psychophysical function, and subjects with the larger exponents in perception are those who exhibit the larger exponents in memory. In addition, a highly positive relation is also evident with the multiplicative constant $\left[r^{2}=.937, F(1,9)=\right.$ $135.48, p<.0001]$ and with the additive constant $\left[r^{2}=\right.$ $.870, F(1,9)=76.91, p<.0001]$. Thus, this close correspondence between memory-and perception-based psychophysical function parameters provides a replication and extension of the second order isomorphism idea established in Shepard and Chipman (1970).

The reperception hypothesis: $A$ critical test. Given that the average exponent for perception is 0.564 , the reperception view requires the memory exponents to be smaller than the perceptual exponents - that is, $0.564^{2}=$ 0.318 , on average. However, in striking contrast to the reperception view, the memory exponent is 0.697 , which is in fact significantly larger than its perceptual counterpart $[t(10)=2.31, p<.043$, by a two-tailed test $]$. This failure of the reperception hypothesis is especially noteworthy because it cannot be attributed to idiosyncratic biases in the manner in which subjects use numbers (e.g., in magnitude estimation) or to differential compression - expansion in the internal psychophysics of number magnitude when memory magnitudes are estimated, as compared with when perceptual magnitudes are estimated (see Krueger, 1989, p. 256, concerning subjective number and the exponent in magnitude estimation).

Although the formulation of the reperception idea has been based exclusively on the exponent of the power function, it is also of interest to provide a fuller characterization of power-function-based memory psychophysics by examining the properties of the multiplicative and the additive constants as well. For perception, the mean of the multiplicative constant is $1.44(S D=3.69$, range $=$ $0.009-12.531)$, and for memory the mean is $0.67(S D=$ 1.39 , range $=0.006-4.625$ ), and these two means do not differ reliably $[t(10)=1.09, p>.30]$. As well, the means for the additive constant are $3.62(S D=4.27$, range $=1.39$ $16.30)$ and $2.51(S D=2.09$, range $=1.31-8.00)$ for perception and memory, respectively, and these means are not reliably different $[t(10)=1.50, p>.16]$.

Relations among the parameters of the power function. Although the primary concern of psychophysicists has been with the exponent of the power function, the multiplicative constant (referred to variously as the measure constant or the coefficient) has also been of both theoretical and empirical interest, as has the relationship between these two parameters. Borg and Marks (1983), while detailing the variety of factors that can influence the size of the measure constant, have strongly encouraged its fuller examination. Of course, Brentano's (1874) formulation of the power function provides a clear and compelling basis for empirical examination of the relationship between the exponent and the multiplicative constant. According to Brentano (see Krueger, 1989, p. 253), letting $k=\Delta I / I$ denote the Weber fraction and $c=\Delta S / S$ constant relative subjective magnitudes, the power law is expressed as

$$
S=I_{0}^{-c / k} I^{c / k},
$$

where $S$ and $I$ denote subjective and physical magnitude, respectively, and the constant, $I_{0}$, the absolute threshold. Denoting the exponent by $E$ and the multiplicative con- 


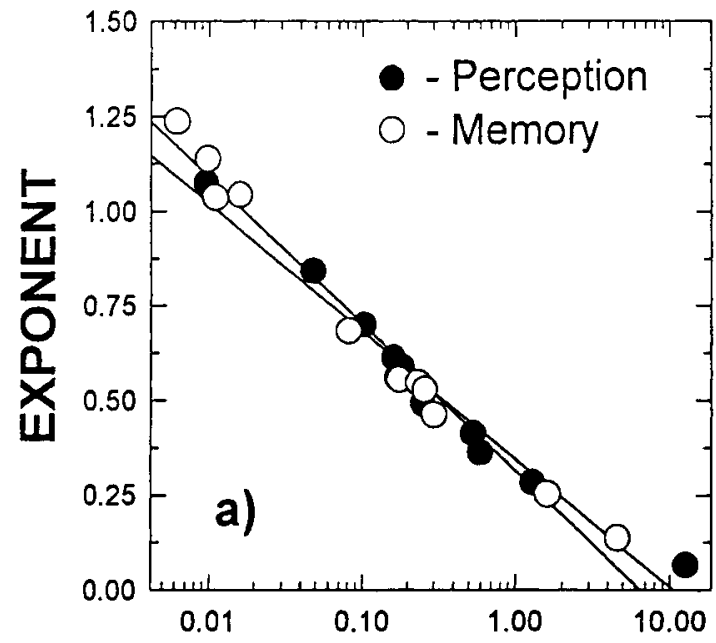

MULTIPLICATIVE CONSTANT

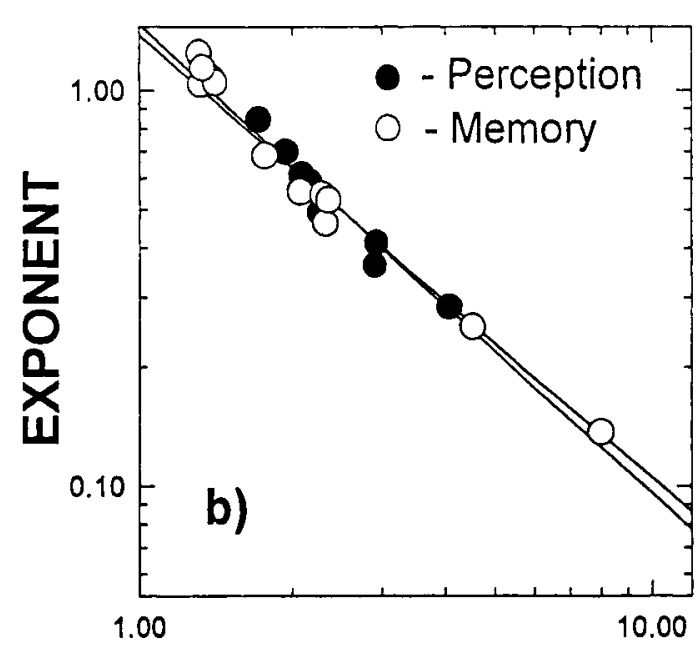

ADDITIVE CONSTANT

Figure 3. Relationship between the exponent and the logarithm of the multiplicative constant in panel a (each point denoting the relationship for an individual subject). Panel b provides the relationship between the logarithm of the exponent and the logarithm of the additive constant. For each case, the relationship is determined separately for perception (filled points) and for memory (unfilled points).

stant by $M$, the relationship between these two parameters is given by

$$
E=\frac{-1}{\log I_{0}} \log M,
$$

and thus, as is evident, the exponent is inversely related to the logarithm of the multiplicative constant $\left(M=I_{0}^{-\mathrm{c} / \mathrm{k}}=\right.$ $I_{0}^{-E}$ ), and the slope of this relationship depends on the absolute threshold, $I_{0}$.

The left panel of Figure 3 provides plots of the relationship between the exponent and the logarithm of the multiplicative constant, and the plots in the right panel characterize the relationship between the exponent and the additive constant, on $\log -\log$ coordinates (i.e., a power function describes the relationship for these two parameters), over subjects, separately for perception and memory.

Regression analyses provide strong support for the Brentano-based relationship between the exponent and the multiplicative constant specified in Equation 3; for perception $\left[r^{2}=.985, F(1,9)=604.01, p<.0001\right]$ and for memory $\left[r^{2}=.983, F(1,9)=447.65, p<.0001\right]$. Furthermore, these linear relationships are virtually identical for perception and memory; the slopes are -0.337 and -0.388 for perception and memory, respectively, adding further force to the view that memory and perception share common properties. However, these analyses of the relationship between the exponent and the multiplicative constant must be viewed cautiously, because Rule (1993) showed that, in the two-parameter power function, the relationship between these two parameters depends on the units used to measure the physical stimuli. Nevertheless, the commonality of the relationship between exponent and multiplicative constant for perception and memory holds independently of Rule's sage theoretical analyses.

The plots in the right panel of Figure 3 show that the value of the exponent is dependent on the value of the additive constant obtained from the iterative routine, as outlined above in the procedure used to obtain the best fitting power function. On $\log -\log$ coordinates, the slopes for perception and memory are -1.113 and -1.180 , respectively, with $r^{2}=.985, F(1,9)=604.01, p<.0001$ for perception, and $r^{2}=.980, F(1,9)=447.65, p<.0001$, for memory. Thus, for both perception and memory and in precisely the same manner for both, as the additive constant increases, the exponent in the power function decreases. Indeed, in the limiting case, as the additive constant becomes arbitrarily large, the exponent goes to zero, which, as Fagot (1963) and Krueger (1989) have noted, is the case in which the power function converges to the highly compressive logarithmic function.

\section{Response Time Analyses}

Ordinal distance effects. Figure 4 shows that RTs with the bilateral quads exhibit a clear ordinal distance effect for both the memorial and the perceptual comparisons. In particular, $\mathrm{RT}(12,23)>\mathrm{RT}(12,24)>\mathrm{RT}(12,25)$, where RT $(12,23)$, for example, denotes the RT, after averaging over the two instructions (i.e., similar/dissimilar), for comparisons with the quad involving pairs 12 and 23 . Similarly, RT $(34,45)>\operatorname{RT}(24,45)>\operatorname{RT}(14,45)$. The plots in Figure 4 were obtained upon averaging RTs with these two different sets of quads.

An ANOVA was conducted with type of comparison (memory vs. perception), whether the middle stimulus in the bilateral quad was Stimulus 2 [with quads of the form 


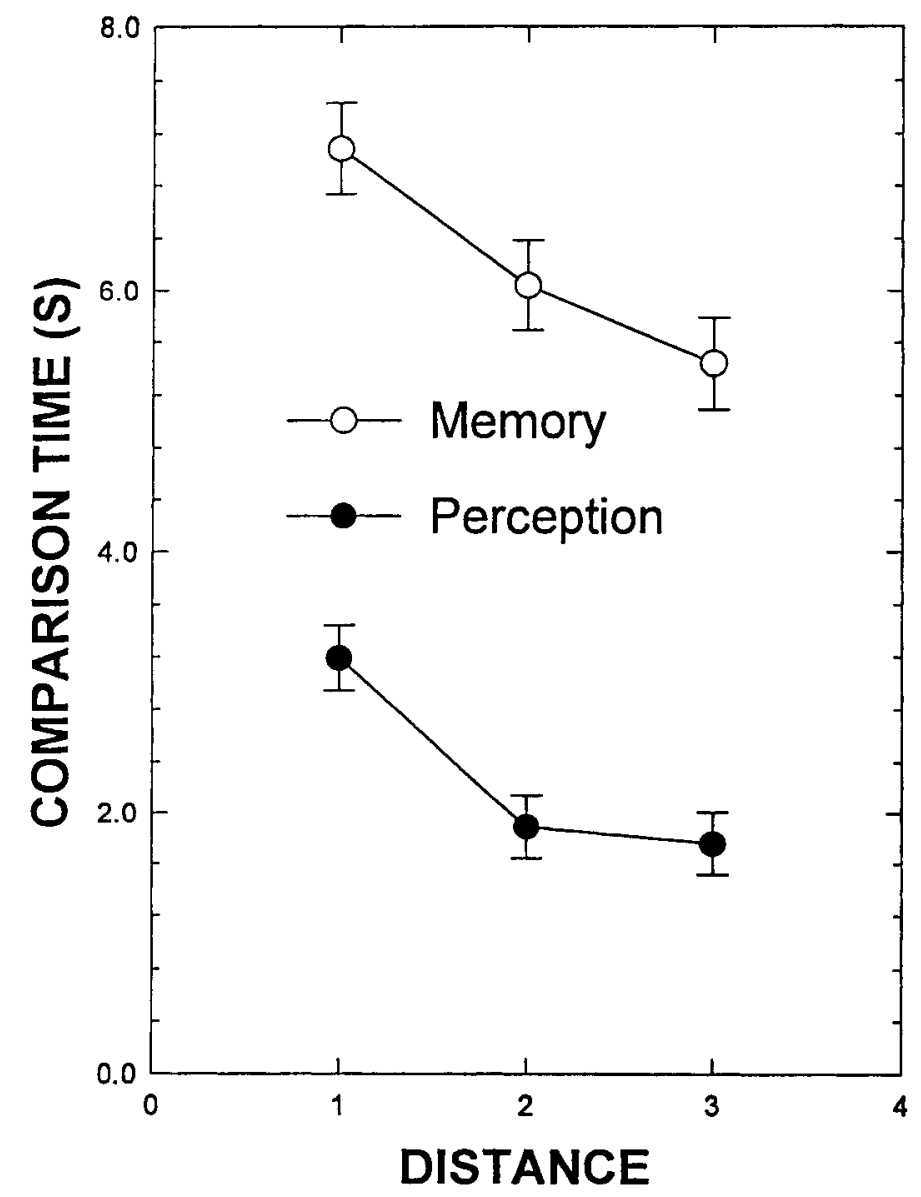

Figure 4. Response times and standard errors as a function of distance for bilateral quads for memory (open circles) and perception (circles). Distance is defined by the difference of the differences in ordinal units. Standard errors are based on the error terms for the distance effect in separate ANOVAs for perception and for memory.

$(12,2 x)$, where $x=3,4,5]$ or Stimulus 4 [with quads of the form $(x 4,45)$, where $x=1,2,3]$, and the instruction (more similar vs. more dissimilar) as within-subjects factors. Throughout, we employ Greenhouse-Geisser adjusted degrees of freedom, although the degrees of freedom indicated are those defined by the design. The main effect of type of comparison was highly reliable $[F(1,10)=$ $40.40, p<.0001$ ], reflecting that (average) RTs were longer with memory-based quads than with perceptually based quads ( 6.18 vs. $2.28 \mathrm{sec}$ ). In addition, establishing a clear parallel with binary relational judgments, RTs with these quaternary relational judgments exhibit a reliable ordinal distance effect $[F(2,20)=9.02, p<.0098]$. No other effects were statistically reliable.

In the above analyses, for these stimuli, ordinal distance and physical distance are perfectly monotonically related; consequently, the dependence of RTs on differences of differences on the basis of the metric properties of the stimuli remains to be established. Nevertheless, the above analyses are important in establishing, in a preliminary manner, further parallels between perceptually based and remembered comparisons, thus extending RT-based memory psychophysics to similarity comparisons. The analyses to be presented in the next section provide an explicit test of the metric-based difference of differences rule, separately for perception and for memory.

Metric-based distance effects. For each subject, for each quad, we first calculated the difference of the difference, $D(i, j, k, l)$, defined by

$$
D(i, j, k, l)=\left\|x_{i}^{\beta}-x_{i}^{\beta}|-| x_{k}^{\beta}-x_{l}^{\beta}\right\|,
$$

where $\beta$ denotes the exponent in the three parameter power function fit to the ALSCAL-based scale values and $x$ the physical value (in pixels) of the stimulus. Least squares linear regression was then used to determine the relationship between RTs and the $D(i, j, k, l)$ s for each subject, separately for perception and memory, with an inverse power law function, $\mathrm{RT}=a D(i, j, k, l)$, with $b<0$, and with the simple linear relationship, $\mathrm{RT}=a D(i, j, k, l)+b$, with $a<0$.

For perception, the average value of $r^{2}$ (over subjects) for the power function fits was 0.497 , with a minimum 
PERCEPTION
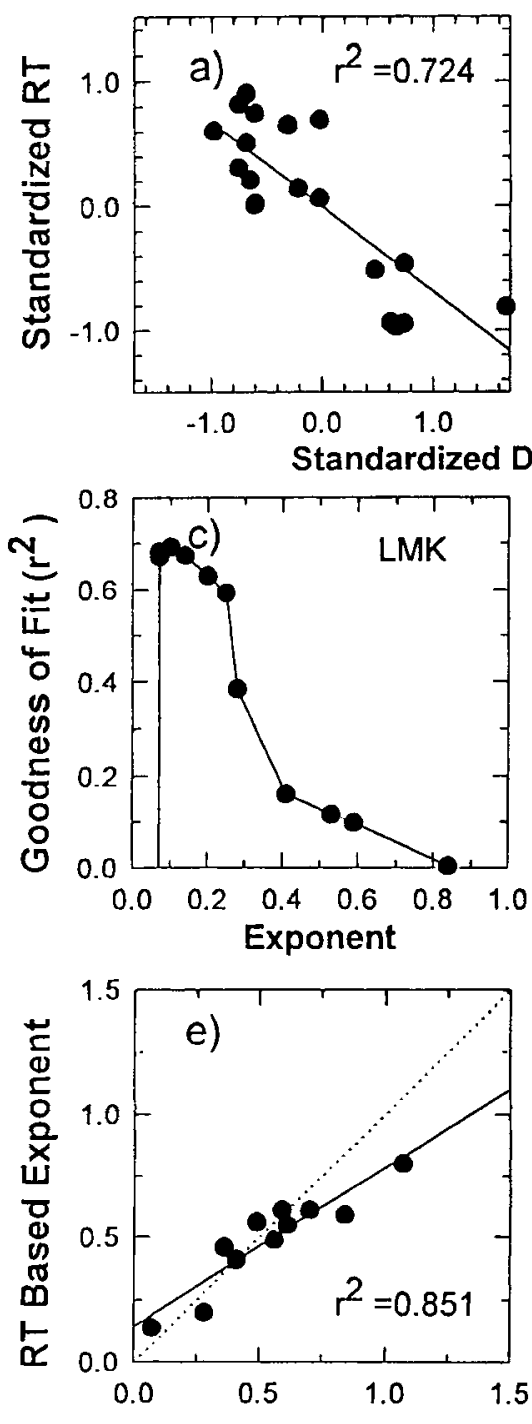

ALSCAL Based Exponent
MEMORY
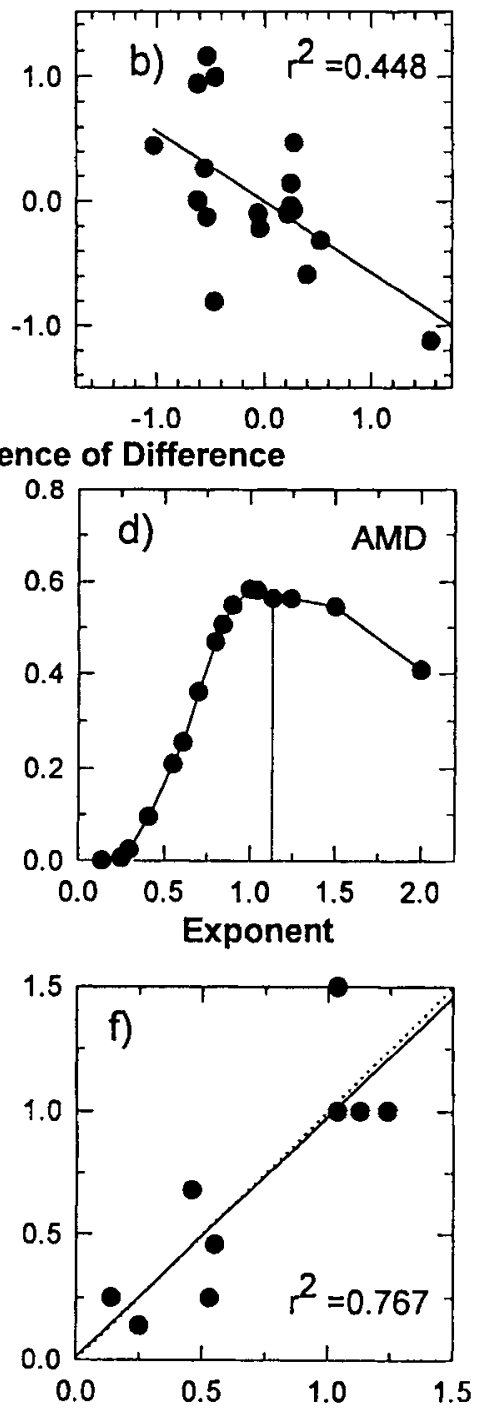

ALSCAL Based Exponent

Figure 5. Mean of standardized response times as a function of the mean of standardized difference of differences for perception (panel a) and for memory (panel b). Goodness of fit $\left(r^{2}\right)$ as a function of the exponent for Subject LMK for perception (panel c) and for memory for Subject AMD (panel d). Drop lines indicate the value of the exponent obtained from the ALSCAL scale values. Relationship between the value of the exponent for the three-parameter psychophysical function obtained from ALSCAL scale values and the value of the exponent corresponding to the maximum value of $\boldsymbol{r}^{2}$ in fitting response times to the difference of differences (each point represents the relationship for a single subject) for perception (panel e) and for memory (panel $f$ ).

value of 0.247 and a maximum of 0.691 . For the linear relationship, the mean value of $r^{2}$ was 0.466 , with $r^{2}$ varying between 0.221 and 0.671 . For memory, the fits were considerably poorer; for the power function, the mean value of $r^{2}$ was 0.161 , with $r^{2}$ varying between 0.005 and 0.414 ; for the linear relationship, $r^{2}$ varied between 0.007 and 0.563 , with a mean value of 0.219 . The fits for 2 subjects, M.M.T. and S.J.M., were extremely poor with both functions (e.g., 0.006 and 0.016 , respectively, for the linear relationship). RTs for these 2 subjects also failed to monotonically decrease with increasing ordinal distance. Thus, these 2 subjects were eliminated from further metric RT analyses. Furthermore, because the fits were as well characterized by the simple linear relationship as the inverse power function, for both perception and memory, the simple linear function was used in subsequent analyses.

To make RTs comparable across subjects, RTs for each subject were standardized by subtracting the mean overall 20 quads and then dividing this difference by the standard deviation. Because the values of $D(i, j, k, l)$ for 
each quad vary widely, depending on the value of $\beta$, the $D(i, j, k, l)$ s were also standardized. Mean standardized RTs are plotted against mean standardized $D(i, j, k, l) \mathrm{s}$ in the top left panel of Figure 5 for perception and in the right for memory.

For the perceptual comparisons, the plot is well characterized by linearity, with a slope of -0.683 (the intercept is necessarily zero) and $r^{2}=.724[t(9)=9.28, p<$ $.001]$. For memory, although the plot is also reliably characterized by linearity, with a slope of -0.567 and $r^{2}=$ $.448[t(7)=3.211, p<.01]$, concomitant with the longer and more variable memory comparisons, deviations from linearity are more evident. Thus, we can conclude that there is support for the difference of differences rule for both perception and memory, in accord with satisfaction of the axioms for a positive-difference structure.

\section{Response Time-Based Estimates of the Exponent of the Psychophysical Function}

For each subject, the obtained RT fits to the $D(i, j, k, l) \mathrm{s}$ were based on the specific value of the exponent, $\beta$, obtained from the fits of the three-parameter power function to the ALSCAL scale values. Strong support that these estimates provide an accurate description of individualsubject psychophysical functions would be obtained if they converge with an entirely independent estimate of the exponent, $\beta$. RTs fit to the $D(i, j, k, l)$ s provide one such estimate. To determine whether these two estimates do, indeed, converge, the exponent, $\beta$, was systematically varied and, for each value of $\beta, D(i, j, k, l)$ was calculated. RTs were then fit as a linear function of the $D(i, j, k, l) \mathrm{s}$, the plot of the goodness of fit index, $r^{2}$, as a function of the exponent was obtained, and the value of the exponent corresponding to the maximal value of $r^{2}$ was determined.

The middle panels of Figure 5 provide examples of the form of these $r^{2}$ versus $\beta$ plots for LMK and AMD, these 2 subjects showing the best fits for perception and memory, respectively. The plots exhibit a well-defined maximum, and the proximity of this maximum to the ALSCALbased estimate of the exponent should be noted. Moreover, this close correspondence between these two independent estimates of the exponent is not confined to these 2 subjects, who happen to have the most orderly RT-D(i,j,k,l) linear relation, as the plots in the bottom panels in Figure 5 show. These plots, with each subject providing a point, of the exponent based on ALSCAL scale values against that obtained from the RT $-D(i, j, k, l)$ relation with the maximal value of $r^{2}$ are well characterized by linearity for both perception $\left[r^{2}=.851, t(9)=18.574\right.$, $p<.001]$ and memory $\left[r^{2}=.762, t(7)=8.812, p<.001\right]$. In summary, these analyses provide powerful converging support for both the ALSCAL-based three-parameter power function, the particular estimate of the exponent, and simultaneously for the differences of differences model for RTs in similarity/dissimilarity comparisons. Such an orderly interlocking of parameters based on the choice and RT data for both perception and memory provides further and very detailed evidence of their commonalties in representations and decisional processing.
Overlapping versus disjoint quads. As indicated earlier, IDS requires that identical order relations must hold with the disjoint quads (e.g., $a b \boldsymbol{S} c d$, for stimuli ordered $a>b>c>d$ ) and the corresponding overlapping quads ( $a c \boldsymbol{S} b d$ ), and strong support was evident for IDS with both the perceptual and the memorial similarity/ dissimilarity judgments. Given this equivalence of relations, it is natural to inquire whether equal decision times are required with the overlapping and the disjoint quads. Further, the support for the difference of differences model, especially at the metric level, motivates closer scrutiny of this model with the disjoint and overlapping quads.

According to the difference of differences model, RTs for the overlapping quads are given by the expression $\mathrm{RT}(a c, b d)=F(d(a, c)-d(b, d))$, where $F$ is a monotone decreasing function and $d(a, c)$ denotes the distance between the representations of stimuli $a$ and $c$. On the assumption of additivity of distances, as in the earlier demonstration that the IDS axiom is necessary, $d(a, c)=$ $d(a, b)+d(b, c)$, and, similarly, $d(b, d)=d(b, c)+d(c, d)$. Consequently, it follows that RT $(a c, b d)=F(d(a, b)-$ $d(c, d))=\mathrm{RT}(a b, c d)$. Thus, the difference of differences model predicts that RTs with the overlapping and the disjoint quads will be identical.

Panel a of Figure 6 provides plots of average RTs with the overlapping and the disjoint quads. An ANOVA was conducted with comparison condition (memory/ perception), type of quad (overlapping/disjoint), and instruction (similar/dissimilar) as within-subjects factors. Overall, comparisons with the memory quads took longer than those with the perceptual quads $[F(1,10)=48.32$, $p<.0001]$. In addition, RTs with the overlapping quads were 817 and $1,323 \mathrm{msec}$ longer than those with the disjoint quads for the perception and memory conditions, respectively $[F(1,10)=29.40, p<.0003]$, but the interaction between comparison condition and type of comparison did not attain conventional statistical significance $[F(1,10)=1.90, p>.195]$. Thus, these findings suggest that a model for similarity/dissimilarity comparisons that asserts that RTs are strictly a function of the difference of the differences in representations is not supported. On the other hand, given that the IDS axiom is strongly supported on the basis of the choices made and given the clear dependence of RT on the difference of the differences, it may well be the case that the RT analyses are sensitive to factors contributing to decisional processing that are not exclusively based on the putative difference of differences, positive-difference structurebased representation.

Semantic congruity effects. We assume that pairs in the disjoint (overlapping) quads are more likely to be viewed as similar (dissimilar), because, generally, pairs in overlapping quads span a wider distance on the underlying continuum than do the pairs in the disjoint quads. Consistent with this view, semantic congruity effects are evident for both perception and memory in the plots in panel $b$ of Figure 6 of the congruity index for the disjoint and the overlapping pairs. The congruity index, obtained 


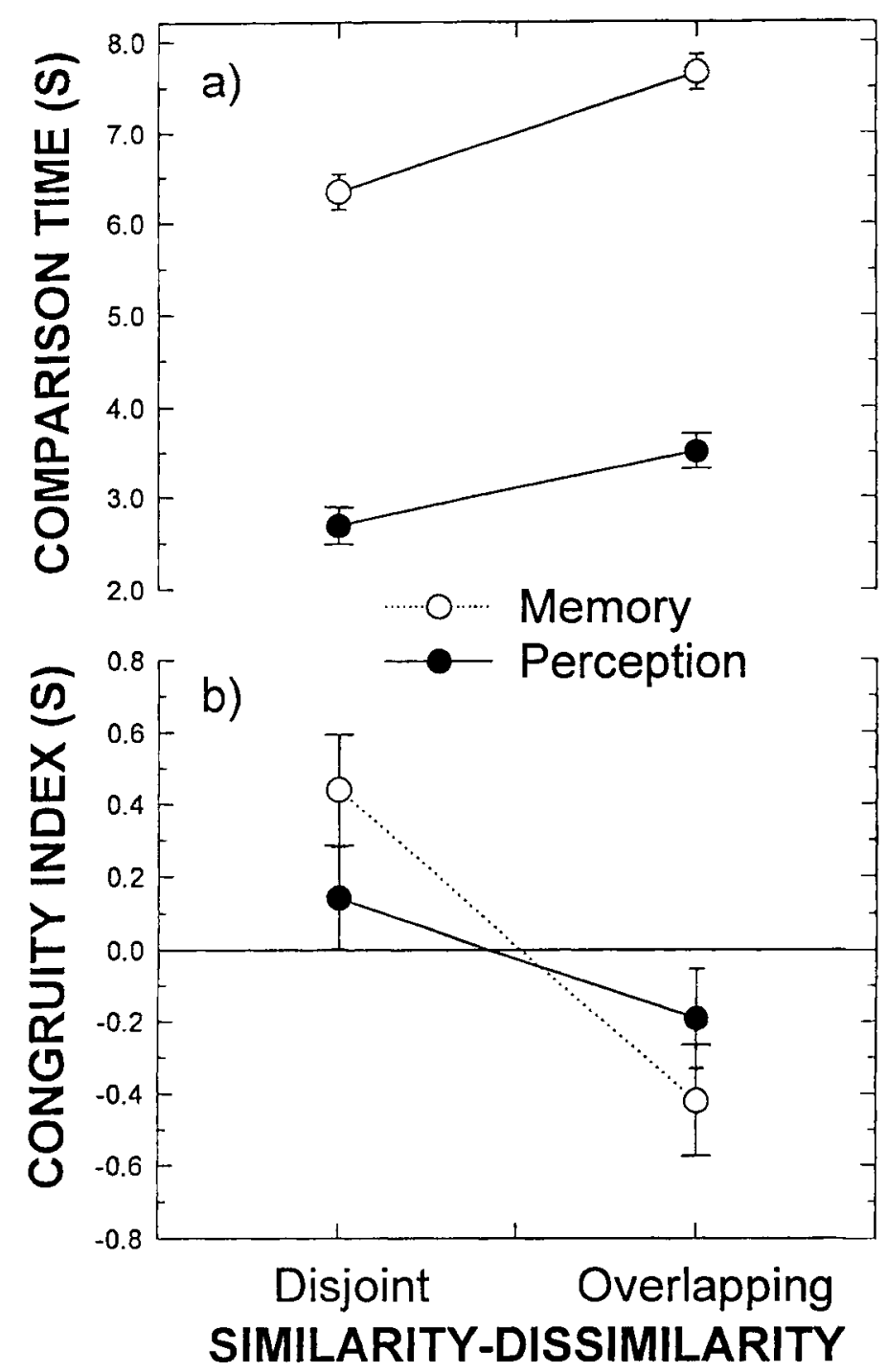

Figure 6. Panel a: Response times (RTs) and standard errors (SEs) for the disjoint and overlapping quads for the perceptual (filled circles) and the remembered (unfilled circles) comparisons. Panel b: RT congruity index and $S E$ s for the disjoint (similar) and overlapping (dissimilar) quads for the perceptual (filled circles) and memory (unfilled circles) conditions. The congruity index is obtained by subtracting mean RTs with the instruction more similar from mean RTs with the instruction more dissimilar, and the $S E$ s are based on the ANOVA with the congruity index. SEs in each plot are based on the error term for the effect of type of quad in separate ANOVAs for perception and for memory.

by subtracting RTs with the instruction more similar from RTs with the instruction more dissimmilar, is positive for the disjoint pairs, reflecting faster RTs with the instruction more similar; for the overlapping pairs, the index is negative, reflecting faster $\mathrm{RTs}$ with the instruction more dissimilar $[F(1,10)=12.96, p<.0048]$. In addition, paralleling an established body of work with binary comparisons (see, e.g., Banks et al., 1982; Petrusic, 1992; Petrusic \& Baranski, 1989; Petrusic et al., 1995), the semantic congruity effect is larger with remembered stimuli; the effect is approximately $165 \mathrm{msec}$ for perception but is over $400 \mathrm{msec}$ for the memory comparisons. This result is supported by a three-way interaction involving type of stimulus, type of quad, and instruction $[F(1,10)=5.05$, $p<.05]$.

\section{GENERAL DISCUSSION}

The present findings extend the area of memory psychophysics to include quaternary relational judgments with remembered magnitudes. Given the satisfaction of the axioms for a positive-difference structure and the low- 
stress-based ALSCAL representations, we conclude that elementary aspects of sensory experience can be maintained in memory at the level of an interval scale. One implication of these findings is that the Moyer and Bayer (1976) range effect, albeit likely to be very small, should be evident, provided that the conditions necessary for its occurrence in perception are ensured. Indeed, Petrusic et al. (1995) report the occurrence of a range effect in their reexamination of this effect, thus implicating analoguebased representations on the basis of binary comparison times. This is not to say that an ordinal coding for remembered magnitudes is never used. In fact, Petrusic et al. have also confirmed that the use of an interval metric versus an ordinal coding is governed by the degree to which the stimulus set is learned. These results, together with the present findings, considerably weaken the hypothesis that remembered magnitudes are exclusively propositionally based, in the form of discrete semantic codes (see, e.g., Banks, 1977; Banks et al., 1982; Cech \& Shoben, 1985).

The reperception hypothesis, developed by Moyer et al. (1978) and Kerst and Howard (1978) in the context of magnitude estimation, is also contradicted by the present findings - namely, that power functions obtained from ALSCAL nonmetric multidimensional representations resulted in exponents for the memory psychophysical function that are not squares of the exponent for the perceptual psychophysical functions. Rather, very closely related structural representations in perception and in memory are implicated, at least in terms of their threeparameter power function characterizations, with, on average, exponents of 0.546 and 0.697 for perception and memory (i.e., both are highly compressive), respectively, ${ }^{6}$ and also with multiplicative and additive constants that are nearly identical.

Our confirmation of an interval scale for remembered magnitudes is consistent with Shepard and Chipman's (1970) view of a second-order isomorphism between perception and memory. In addition, our results complement recent suggestions of a common representation for perceived and remembered odors by Algom and Cain (1991) and the findings of Izmailov and Sokolov (1992), who demonstrated that color memory exhibits the structures evident in perception only after sufficient paired-associate training. Although we have found evidence for an interval scale for remembered magnitudes, its theoretical basis remains to be established, and we do not speculate about its nature (e.g., whether imagery is involved or whether the representation can arise from a highly articulated propositional network) at this time.

Finally, the present findings revealed substantially faster comparisons for disjoint than for overlapping quads with both memorial and perceptual comparisons. As mentioned, on the face of it, this result is inconsistent with a difference of differences decision model of similarity/ dissimilarity comparisons. However, given that the axioms for a positive-difference structure were satisfied and the convergence of the ALSCAL-based estimate of the exponent in the power function with that obtained with the fits of RTs to the $D(i, j, k, l) \mathrm{s}$, the additional time re- quired with the overlapping comparisons might not arise as a failure of the difference of differences rule but, rather, as a consequence of the time required to cancel out the common distance, thereby effectively reducing overlapping quads to disjoint ones.

In conclusion, our fundamental measurement analyses of quaternary relational judgments confirmed an interval metric scale for (well-learned) remembered magnitudes. The question of how people perform the complex quaternary judgment was addressed through RT analyses. The RT analyses established that the various RT relations evident with direct perceptual stimuli were also evident with remembered magnitudes. However, the full set of RT relations could not be accounted for by the difference of differences model, even though this model was supported through the tests of the axioms for a positivedifference structure and its fits with the RTs. Clearly, more detailed theoretical development and further experimental work are needed to resolve this problem in the psychophysics of memory.

\section{REFERENCES}

ALGoM, D. (1992). Memory psychophysics: An examination of its perceptual and cognitive prospects. In D. Algom (Ed.), Psychophysical approaches to cognition (pp. 441-513). Amsterdam: Elsevier.

Algom, D., \& CAIN, W. (1991). Remembered odors and mental mixtures: Tapping reservoirs of olfactory knowledge. Journal of Experimental Psychology: Human Perception \& Performance, 17, 1104-1119.

Algom, D., Wolf, Y., \& BERGMAN, B. (1985). Integration of stimulus dimensions in perception and memory: Composition rules and psychophysical relations. Journal of Experimental Psychology: General, 114, 451-471.

BANKS, W. P. (1977). Encoding and processing of symbolic information in comparative judgments. In G. H. Bower (Ed.), The psychology of learning and motivation (Vol. 11, pp. 101-159). New York: Academic Press.

Banks, W. P., Mermelstein, R., \& Yu, H. K. (1982). Discriminations among perceptual and symbolic stimuli. Memory \& Cognition, 10, 265-278.

Baranski, J. V., \& Petrusic, W. M. (1992). The discriminability of remembered magnitudes. Memory \& Cognition, 20, 254-270.

BeALS, R., KRANTZ, D. H., \& TVERSKy, A. (1968). Foundations of multidimensional scaling. Psychological Review, 75, 127-142.

BJörkman, M., Lundberg, U., \& TARnblom, S. (1960). On the relationship between percept and memory: A psychophysical approach. Scandinavian Journal of Psychology, 1, 136-144.

BLOCK, H. D., \& MARsChaK, J. (1960). Random orderings and stochastic theories of responses. In I. Olkin, S. Ghurye, W. Hoeffding, W. Madow, \& H. Mann (Eds.), Contributions to probability and statistics (pp. 97-132). Stanford: Stanford University Press.

BorG, G. A. V., \& MARKs, L. E. (1983). Twelve meanings of the measure constant in psychophysical power functions. Bulletin of the Psychonomic Society, 21, 73-75.

BradteY, D., \& Vido, D. (1984). Psychophysical functions for perceived and remembered distance. Perception, 13, 315-320.

Brentano, F. (1874). Psychologie vom empirischen Standpunkte. Leipzig: Duncker \& Humblot.

CECH, C., \& SHOBEN, E. (1985). Context effects in symbolic magnitude comparisons. Journal of Experimental Psychology: Learning, Memory, \& Cognition, 11, 299-315.

Сech, C., Shoben, E., \& Love, M. (1990). Multiple congruity effects in judgments of magnitude. Journal of Experimental Psychology: Learning. Memory, \& Cognition, 16, 1142-1152.

Chew, E. I., \& Richardson, J. T. E. (1980). The relationship between perceptual and memorial psychophysics. Bulletin of the Psvchonomic Society, 16, 25-26. 
CoomBs, C. H. (1952). A theory of psychological scaling. Engineering Research Institute Bulletin (No. 34). Ann Arbor: University of Michigan Press.

CoOMBS, C. H. (1964). A theory of data. New York: Wiley.

DEMBER, W. N. (1957). The relation of decision-time to similarity. Jour nal of Experimental Psychology, 53, 68-72.

FAGOT, R. F. (1963). On the psychophysical law and estimation procedures in psychophysical scaling. Psychometrika, 28, 145-160.

FECHNER, G. T. (1966). Elements of psychophysics (H. E. Adler, Trans.) New York: Holt, Rinehart \& Winston. (Original work published 1860)

HARTLEY, A. (1977). Mental measurement in the magnitude estimation of length. Journal of Experimental Psychology: Human Perception \& Performance, 3, 622-628.

HENDERSON, J. M., \& WELL, A. D. (1985). Symbolic comparisons with and without perceptual referents: Is interval information used? Memory \& Cognition, 13, 176-182.

Izmailov, C. A., \& Sokolov, E. N. (1992). A semantic space of color labels. Psychological Science, 3, 105-110.

KENNEDY, R. (1990). Psychometric functions with remembered standards: Weber fractions and serial position effects. Unpublished master's thesis, Carleton University.

KERST, S. M., \& Howard, J. H. (1978). Memory psychophysics for visual area and length. Memory \& Cognition, 6, 327-335.

KrantZ, D. H., LuCE, R. D., SupPes, P., \& TVersky, A. (1971). Foundations of measurement (Vol. 1). New York: Academic Press.

KRUEGER, L. E. (1989). Reconciling Fechner and Stevens: Toward a unified psychophysical law. Behavioral \& Brain Sciences, 12, 251-320.

KRUSKAL, J. B. (1964). Non metric multidimensional scaling: A numerical method. Psychometrika, 29, 115-129.

MOYER, R. S. (1973). Comparing objects in memory: Evidence suggesting an internal psychophysics. Perception \& Psychophysics, 13, 180-184.

MOYER, R. S., \& BAYER, R. H. (1976). Mental comparison and the symbolic distance effect. Cognitive Psychology, 8, 228-246.

Moyer, R. S., Bradley, D. R., Sorensen, M. H., Whiting, J. C., \& MANSFIELD, D. P. (1978). Psychophysical functions for perceived and remembered size. Science, 200, 330-332.

Moyer, R. S., SkLAREW, P., \& Whiting, J. C. (1982). Memory psychophysics. In H. G. Geissler \& P. Petzold (Eds.), Psychophysical judgment and the process of perception (pp. 35-46). Berlin: VEB Deutscher Verlag der Wissenschaften.

Nelson, T. O., \& Chaiklin, S. (1980). Immediate memory for spatial location. Journal of Experimental Psychology: Human Learning \& Memory, 6, 529-545.

Parker, S., SChNeIder, B., \& Kanow, G. (1975). Ratio scale measurement of the perceived length of lines. Journal of Experimental Psychology: Human Perception \& Performance, 104, 195-204.

PeTRUSIC, W. M. (1992). Semantic congruity effects and theories of the comparison process. Journal of Experimental Psychology: Human Perception \& Performance, 18, 962-986.

Petrusic, W. M., \& Baranski, J. V. (1989). Semantic congruity effects in perceptual comparisons. Perception \& Psychophysics, 45, 439-452.

Petrusic, W. M., Baranski, J. V., \& Aubin, P. (1995, November). Comparative judgments with perceived and remembered magnitudes. Paper presented at the 36th Annual Meeting of the Psychonomic Society, Los Angeles.

Petrusic, W. M., \& Jamieson, D. G. (1979). Resolution time and the coding of arithmetic relations on supraliminally different visual extents. Journal of Mathematical Psychology, 19, 89-107.

Petrusic, W. M., \& Jamieson, D. G. (1989). Comparative judgments of the ease of sameness-difference judgment. American Journal of Psychology, 102, 69-90.

RULE, S. J. (1993). Analyzing coefficients of psychophysical power functions. Perception \& Psychophysics, 54, 439-445.

Schneider, B., Parker, S., \& Stein, D. (1974). The measurement of loudness using direct comparisons of sensory intervals. Journal of Mathematical Psychology, 11, 259-273.

SHEPARD, R. N. (1966). Metric structures in ordinal data. Journal of Mathematical Psychology, 3, 287-315.

ShePard, R. [N.], \& Chipman, S. (1970). Second-order isomorphism of internal representations: Shapes of states. Cognitive Psychology, 1, 1-17.
SPSS INC. (1988). SPSS-X user's guide (3rd ed.). Chicago: Author. Stevens, S. S. (1975). Psychophysics: Introduction to its perceptual, neural and social prospects. New York: Wiley.

Stevens, S. S., \& Galanter, E. H. (1957). Ratio scales and category scales for a dozen perceptual continua. Journal of Experimental Psychology, 54, 377-411.

SUPPES, P., \& WINET, M. (1955). An axiomatization of utility based on the notion of utility differences. Management Science, 1, 259-270.

SUPPES, P., \& ZINNES, J. L. (1963). Basic measurement theory. In R. D. Luce, R.R. Bush, \& E. Galanter (Eds.), Handbook of mathematical psychology. (Vol. 1, pp. 1-76). New York: Wiley.

TaKane, Y., YounG, F. W., \& DE LeEUW, J. (1977). Nonmetric individual differences multidimensional scaling: An alternating least squares method with optimal scaling features. Psychometrika, 42, 7-67.

Teghtsoonian, M., \& Teghtsoonian, R. (1965). Seen and felt length. Psychonomic Science, 3, 465-466.

TeghtsoOnian, R. (1973). Range effects in psychophysical scaling and a revision of Stevens' law. American Journal of Psychology, 86, 3-27.

\section{NOTES}

1. Interestingly, this latter case is indistinguishable from the uncertainty hypothesis (see, e.g., Kerst \& Howard, 1978), which asserts that uncertainty always accompanies the retrieval of quantitative information from memory and invariably leads to an unwillingness to render extreme judgments. Consequently, a restriction in the response range occurs, and, with this restricted range, the exponent necessarily decreases. Alternatively, Algom, Wolf, and Bergman (1985) venture that the greater uncertainty in memory results in an increase in the "effective" stimulus range. Appealing to the general principle established by $R$. Teghtsoonian (1973) that the exponent and stimulus range are inversely related, it follows that the exponent will be smaller in the memory case than in the perceptual case.

2. We also use the notation $a b D c d$ to denote the judgment that the pair $a b$ is more dissimilar than the pair $c d$ and, generally, $a b D c d$ iff $c d \boldsymbol{S} a b$.

3. Petrusic and Jamieson (1979) provided a test of the positivity axiom through an analysis of RTs with comparative judgments (e.g., which of two triangles is taller). In particular, for all triples ordered $a>$ $b>c$ on the underlying continuum, they showed that RT $(a b)>\mathrm{RT}(a c)$ and $\mathrm{RT}(b c)>\mathrm{RT}(a c)$

4. The nontestable, technical, axioms are: (1) solvability, a structural condition that states that the intervals are sufficiently dense so that any interval can be copied within any larger interval, and (2) Archimedean, which assumes the existence of a finite standard sequence such that end to end concatenation of adjacent elements in the sequence ensures standard (arbitrarily fine) intervals that can serve as units. Any interval can thus be measured in terms of the number of copies of this unit interval.

5 . In order to see the relationship between weak monotonicity and intradimensional subtractivity, consider the following premise: if $a b S b c$ and $b c \boldsymbol{S} c d$. Weak monotonicity implies $a c S b d$, and transitivity $a b S c d$. Thus, IDS follows-that is, $a b \boldsymbol{S} c d$ if and only if $a c \boldsymbol{S} b d$. In the present experiment, in order to keep the task manageable, only five stimuli were in the set. Consequently, the weak monotonicity axiom could not be tested fully. Rather, IDS was tested.

6. When magnitude estimation is used, the exponent for visual length is invariably near 1.0 (see, e.g., Hartley, 1977; Stevens \& Galanter, 1957; M. Teghtsoonian \& R. Teghtsoonian, 1965).The value of 0.55 obtained in the present experiment with similarity comparisons converges nicely with the 0.50 obtained by Parker, Schneider, and Kanow (1975), who required magnitude estimates of the similarity of pairs of visual extents in one experiment and category ratings of similarity in another. In their experiment, as in the present, a nonmetric MDS routine was used to obtain the representations. If the magnitude estimation-based exponents are multiplied by the exponent for subjective number, exponents in the range obtained here and by Parker et al. are obtained (cf. Krueger, 1989).

(Manuscript received September 23, 1996; revision accepted for publication July 17, 1997.) 